\title{
Rigidity of harmonic measure
}

\author{
by
}

\section{P o povici and A. Volberg (East Lansing, Mich.)}

\begin{abstract}
Let $J$ be the Julia set of a conformal dynamics $f$. Provided that $f$ is polynomial-like we prove that the harmonic measure on $J$ is mutually absolutely continuous with the measure of maximal entropy if and only if $f$ is conformally equivalent to a polynomial. This is no longer true for generalized polynomial-like maps. But for such dynamics the coincidence of classes of these two measures turns out to be equivalent to the existence of a conformal change of variable which reduces the dynamical system to another one for which the harmonic measure equals the measure of maximal entropy.
\end{abstract}

1. Introduction. The harmonic measure in a dynamical context appeared for the first time in Brolin's paper $[\mathrm{Br}]$ where it was established that the harmonic measure $\omega$ associated with the unbounded Fatou component $A_{\infty}(f)$ of a polynomial $f$ is balanced, which means that its Jacobian is constant. Later when the ergodic theory of rational maps was developed by Lyubich [Ly1], [Ly2] and independently by Freire, Lopes and Mañé [FLM], $[\mathrm{M}]$, this balanced measure was interpreted as the unique measure $m$ of maximal entropy.

When we have a dynamical system more general than polynomial, the natural question of comparison of these two measures arises. For rational $f$ it was considered by Lopes in [Lo], where it was proved that if $\infty \in \mathbb{C} \backslash J(f)$ is a fixed point of $f$, then it follows from $m=\omega$ that $f$ is a polynomial. We will consider the local setting of the problem when $f$ is defined only on a neighborhood of an invariant compact set $J_{f}$. The question is to characterize the situation when $\omega \approx m$, where " $\approx$ " denotes mutual absolute continuity. It certainly happens when $f$ is conformally equivalent to a polynomial. In this paper we discuss the converse problem in the case when $f$ is a generalized polynomial-like map (GPL).

Let us pass to precise definitions. Let $U, U_{1}, \ldots, U_{k}$ be $k+1$ topological discs with real analytic boundaries such that $\bar{U}_{i} \subset U, i=1, \ldots, k$, and $\bar{U}_{i} \cap \bar{U}_{j}=\emptyset, i \neq j$. Consider a map $f: \bigcup_{i=1}^{k} U_{i} \rightarrow U$ which is a branched

1991 Mathematics Subject Classification: 30D05, 58F23.

Research of the second author partially supported by NSF Grant DMS 9302728. 
covering of degree $d_{i}<\infty$ on each $U_{i}$. Set $K_{f}=\bigcap_{n \geq 0} U^{n}$, where $U^{n}=$ $f^{-n}(U)$. We call $J_{f}=\partial K_{f}$ the Julia set of $f$. It is also the boundary of $A_{\infty}=A_{\infty}(f)=\overline{\mathbb{C}} \backslash K_{f}$. The degree of $f$ is $d=d_{1}+\ldots+d_{k}$. Let us say that $f$ is polynomial-like (in the sense of Douady and Hubbard [DH]) if $k=1$ and $d=d_{1} \geq 2$.

Saying that two maps $f, g$ are (conformally) conjugate we mean that there is a (conformal) conjugation in some neighborhoods of the Julia sets. Any GPL is quasiconformally conjugate to a polynomial, and pulling back the measure of maximal entropy of this polynomial we obtain $m=m_{f}$, the measure of maximal entropy for $f$. In this paper, a GPL $g$ is called maximal if $\omega_{g}=m_{g}$, where $\omega_{g}$ denotes the harmonic measure evaluated at infinity. As infinity has no special role for GPL we introduce the following class of GPL.

We call $f$ conformally maximal if there exists another GPL $g$ which is maximal and if there exists a conformal map $h: U_{f} \rightarrow U_{g}$ which conjugates the dynamics: $f=h^{-1} \circ g \circ h$.

In this paper we prove the following theorems. lent:

Theorem 1. Let $f$ be a GPL. Then the following assertions are equiva-

1. $f$ is conformally maximal.

2. $\omega_{f} \approx m_{f}$.

THEOREM 2. Let $f$ be a polynomial-like map. The following are equivalent:

1. $f$ is conformally conjugate to a polynomial.

2. $\omega_{f} \approx m_{f}$.

To prove these results we will use the technique of $[\mathrm{LyV}],[\mathrm{BV}]$ and the following result proved by the first author:

TheOREM 3. Let $f$ be a GPL. Then there exists a finite measure $\nu$ on $J_{f}$ such that $\nu$ is $f$-invariant and $\nu \approx \omega_{f}$.

Theorem 3 was proved independently by A. Zdunik [Z2]. She also derived Theorems 1 and 2 from it. We give here our very short proof of Theorem 3.

Theorems 1 and 2 were proved by Z. Balogh [B] in the case when $J_{f}$ is totally disconnected without any assumptions of hyperbolicity. His proof is very different from the one here or from Zdunik's proof (which also differs from ours). In particular, Balogh does not need to construct the invariant harmonic measure (Theorem 3).

Let us mention that the results of Theorems 1, 2 and 3 were stated as conjectures in $[\mathrm{LyV}],[\mathrm{BV}]$ and $[\mathrm{BPV}]$, where particular cases were proved (e.g. semihyperbolic case in $[\mathrm{BV}])$. 
2. Existence of invariant harmonic measure. We will use the following result of Y. N. Dowker and A. Calderón which we found in [Fo]. We formulate it in a convenient form.

Theorem A. Let $\mu$ be a probability measure on a compact set $X$. Let $T: X \rightarrow X$ be a continuous endomorphism such that $\mu$ is completely nonsingular with respect to $T$. Then there exists a $T$-invariant probability measure $\lambda$ absolutely continuous with respect to $\mu$ if and only if

$$
\mu(E)<1 \Rightarrow \sup _{n} \mu\left(f^{-n} E\right)<1 .
$$

If $\mu$ is ergodic then $\lambda$ is ergodic.

To use Theorem A for $X=J_{f}$ and $\mu=\omega_{f}$ we are going to estimate $\omega\left(f^{-n} E\right)$ via $\omega(E)$.

Fix an arbitrary Borel set $E \subset J$ with $\omega(E)=1-\varepsilon<1$. Let $\Gamma$ be a smooth curve encircling $J$ and separating it from $\partial U$ and let $\Gamma^{n}=f^{-n}(\Gamma)$. The main things now are six notations.

Let $\omega$ and $v$ denote the harmonic measures of $E$ with respect to $A_{\infty}(f)$ and $U \backslash K_{f}$ respectively. Let $V$ and $W$ denote the harmonic measures of $J \backslash E$ with respect to $A_{\infty}$ and $U \backslash K_{f}$ respectively. For any function $\phi$ let $\phi^{n}$ denote $\phi \circ f^{n}$ where defined. As usual, $\omega_{\Omega}(S, z)$ denotes the harmonic measure of $S$ evaluated at $z$ with respect to $\Omega$.

First we need a simple lemma. Fix a compact set $K$ in $\Omega$ and consider two harmonic measures on $\partial \Omega$ with respect to $\Omega$ and with respect to $\Omega \backslash K$, evaluated at the same point $a \in \Omega \backslash K$.

Lemma 2.1. The two harmonic measures on $\partial \Omega$ are boundedly equivalent.

Proof. We present the proof in the case when all points of $\partial \Omega$ are regular. Only this case is used in what follows. Let $O$ be a neighborhood of $\partial \Omega$ with smooth boundary and satisfying $O \cap(K \cup\{a\})=\emptyset$. Let $G$ and $g$ be Green's functions of $\Omega$ and $\Omega \backslash K$ respectively, with pole at $a$. Then by Harnack's principle, $c G \leq g \leq G$ on the boundary of $O$. By the maximum principle this inequality extends to $O$ (both functions vanish on $\partial \Omega$ ). Then clearly the functions $g-c G$ and $G-g$ are subharmonic in $O$ and so their Riesz measures are nonnegative. The Riesz measures of $G$ and $g$ being equal to our harmonic measures, we are done.

Proof of Theorem 3 . We have to prove that

$$
\omega_{A_{\infty}}(E, \infty) \leq 1-\varepsilon \Rightarrow \omega_{A_{\infty}}\left(f^{-n} E, \infty\right) \leq 1-\delta .
$$

As $\omega(\infty) \leq 1-\varepsilon$, we get $W(\infty) \geq \varepsilon$. Then $W(\xi) \geq c \varepsilon$ on $\Gamma$ and by Lemma 2.1, $V(\xi) \geq \delta$ on $\Gamma$. Then $V^{n}(\xi) \geq \delta$ on $\Gamma^{n}$. But these functions vanish on $\partial U^{n}$ and so $\omega_{A_{\infty}}\left(f^{-n}(J \backslash E), \xi\right) \geq \delta$ on $\Gamma^{n}$. So $\omega_{A_{\infty}}\left(f^{-n} E, \xi\right) \leq$ 
$1-\delta$ on $\Gamma^{n}$. As $\Gamma^{n}$ separates $J$ from $\infty$ we get $\omega\left(f^{-n} E\right)=\omega_{A_{\infty}}\left(f^{-n} E, \infty\right)$ $\leq 1-\delta$.

We are going to prove that the just constructed invariant harmonic measure $\nu$ is boundedly equivalent to $\omega$.

THEOREM 4. There exist constants $0<c_{1}, c_{2}<\infty$ such that

$$
c_{1} \leq d \nu / d \omega \leq c_{2} .
$$

Proof. Let us prove first the right inequality. We wish to repeat the above considerations but it seems hard to get rid of the influence of $\partial U$. However, we are going to prove that $\omega(E) \leq \varepsilon \Rightarrow \omega\left(f^{-n} E\right) \leq c \varepsilon$ for a certain finite $c$. The construction of an invariant measure in [Fo] then gives the right inequality. Let us fix $\Gamma$ as above and such that

$$
\max _{\xi \in \Gamma} \omega_{U \backslash K_{f}}(\partial U, \xi) \leq 1 / 2 .
$$

Then if $\Omega_{n}$ is any component of $U^{n}$ and $\gamma_{n}=\Gamma^{n} \cap \Omega_{n}$, we can write $\omega_{U \backslash K_{f}}\left(\partial U, f^{n}(\xi)\right)=\omega_{\Omega_{n} \backslash K_{f}}\left(\partial \Omega_{n}, \xi\right)$ and thus

$$
\omega_{\Omega_{n} \backslash K_{f}}\left(\partial \Omega_{n}, \xi\right) \leq 1 / 2, \quad \xi \in \gamma_{n} .
$$

We start with the chain of implications:

$$
\begin{aligned}
\omega(\infty) \leq \varepsilon & \Rightarrow \omega(\xi) \leq C_{\Gamma} \varepsilon \text { for } \xi \in \Gamma \\
& \Rightarrow v(\xi) \leq C_{\Gamma} \varepsilon \text { for } \xi \in \Gamma \Rightarrow v^{n}(\xi) \leq C_{\Gamma} \varepsilon \text { for } \xi \in \gamma_{n} .
\end{aligned}
$$

Let us compare $u_{1}(\xi)=v^{n}(\xi)$ with $u_{2}(\xi)=\omega_{A_{\infty}}\left(f^{-n} E, \xi\right)$ on $\gamma_{n}$ for each component $\Omega_{n}$ of $U^{n}$. By the Poisson formula in $\Omega_{n} \backslash K_{f}$ we have

$$
u_{2}(\xi)-\int_{\partial \Omega_{n}} u_{2}(\eta) d \omega_{\Omega_{n} \backslash K_{f}}(\eta, \xi)=u_{1}(\xi) .
$$

Let $u_{2}\left(\xi_{0}\right)=\max _{\gamma_{n}} \max _{\xi \in \gamma_{n}} u_{2}(\xi)=\max _{\xi \in \Gamma^{n}} u_{2}(\xi)$. Then by (2.1) we have $u_{2}\left(\xi_{0}\right)(1-1 / 2) \leq u_{1}\left(\xi_{0}\right) \leq C_{\Gamma} \varepsilon$. But $\Gamma^{n}$ separates $J_{f}$ from $\infty$ and so

$$
\omega_{A_{\infty}}\left(f^{-n} E, \infty\right)=u_{2}(\infty) \leq 2 C_{\Gamma} \varepsilon
$$

The left inequality can be proved in exactly the same way.

\section{Automorphic harmonic function}

THEOREM 1. Let $f$ be a GPL. Then the following assertions are equivalent:

1. $f$ is conformally maximal.

2. $\omega_{f} \approx m_{f}$.

Proof. We will be using the following notations: if $u$ is a subharmonic function then $\mu_{u}=\Delta u$ is its Riesz measure. Let $G$ be Green's function of $A_{\infty}(f)$ with pole at $\infty$. We know that $\mu_{G}=\omega$. Let $\Phi=d \mu_{G \circ f} / d \mu_{G}$ and 
$\phi=\log \Phi$. Clearly $\Phi$ is bounded away from zero and infinity (this is just Harnack's inequality essentially). It will be important for us that $\Phi$ is the Jacobian of $\omega$ with respect to $f$. Let $\varrho=d \nu / d \omega$ and $\gamma=\log \varrho$. The measures $\nu$ and $m$ are finite and invariant and $m$ is ergodic. So $\nu \approx m$ implies $\nu=m$. We start with the homology equation:

$$
\phi-\log d=\gamma \circ f-\gamma \quad \omega \text {-a.e. on } J .
$$

This is obvious from the computation of the Jacobians of the measures $\nu=m$.

To prove our result it is sufficient (and necessary) to construct an automorphic harmonic function $\tau$ ([LyV], $[\mathrm{BV}]$ and $[\mathrm{BPV}]$ ), i.e. a function satisfying the following:

1) $\tau$ is subharmonic in $U, \tau \geq 0$,

2) $\tau$ vanishes on $K_{f}, \tau>0$ on $U \backslash K_{f}$,

3) $\tau$ is harmonic in $U \backslash K_{f}$,

4) $\tau(f z)=d \tau(z)$.

To construct such a function we first construct a disc $B=B(x, r)$ centered at the Julia set and a nonnegative subharmonic function $u$ in $B$ such that

$$
d \omega / d \mu_{u}=e^{\gamma} \quad \text { on } B .
$$

Let $F$ with $\omega(F)>0$ be a set on which $\gamma$ is continuous. Let $F_{0} \subset F$ with $\omega\left(F_{0}\right)>0$ be such that

$$
\lim _{\varepsilon \rightarrow 0} \frac{\omega(B(y, \varepsilon) \cap F)}{\omega(B(y, \varepsilon))}=1 .
$$

We use the natural extension $(\widetilde{J}, \widetilde{f}, \widetilde{m})$ of $(J, f, m)$. Here $\widetilde{f}$ is just the left shift. We denote by $\pi: \widetilde{J} \rightarrow J$ the projection onto the " 0 " coordinate. Then $\widetilde{m}\left(\pi^{-1}\left(F_{0}\right)\right)>0$ and by the ergodicity of $\widetilde{m}$ one can choose $\widetilde{x}$ such that $\widetilde{f}^{-n}(\widetilde{x}) \in \pi^{-1}\left(F_{0}\right)$ with positive frequency. In particular, we have chosen $x \in J_{f}$ and the sequence of compatible inverse images $x_{n}$ of $x$ such that $x_{n} \in F_{0}$ with positive frequency. But one can do more (see [FLM], [Z1]): we can choose $B=B(x, r)$ such that on $3 B$ there are univalent compatible inverse branches $F_{n}$ such that

$$
\begin{gathered}
\operatorname{diam} F_{n}(2 B) \leq e^{-n \delta}, \\
x_{n}=F_{n}(x) \text { meets } F_{0} \text { with positive frequency, }
\end{gathered}
$$

Let us consider the family $u_{n}=d^{n} G \circ F_{n}$ in $3 B$. Then by (3.1),

$$
\frac{d \omega}{d \mu_{u_{n}}}(y)=e^{\gamma(y)-\gamma\left(F_{n}(y)\right)}, \quad y \in J_{f} \cap 3 B .
$$


In particular, $\left\|\mu_{u_{n}}\right\| \leq C<\infty$ and moreover $c_{1} \omega \leq \mu_{u_{n}} \leq c_{2} \omega$ (see Theorem 4 which gives the boundedness of $\gamma)$.

Let $\left\{n_{k}\right\}$ be a subsequence such that $\gamma\left(x_{n_{k}}\right) \rightarrow c$ as $k \rightarrow \infty$, and $x_{n_{k}} \in$ $F_{0}$. Without loss of generality we can think that

$$
\left|\gamma\left(x_{n_{k}}\right)-c\right| \leq 2^{-k}, \quad \frac{\omega\left(F_{n_{k}}(2 B) \backslash F\right)}{\omega\left(F_{n_{k}}(2 B)\right)} \leq 2^{-k} .
$$

The last assertion follows from (3.3), (3.4). Let $E_{k}=F_{n_{k}}^{-1}\left(F \cap F_{n_{k}}(2 B)\right)$. Then

$$
\begin{gathered}
\left|d \omega / d \mu_{n_{k}}-e^{\gamma-c}\right| \leq C_{1} 2^{-k} \quad \text { on } E_{k}, \\
\mu_{n_{k}}\left(2 B \backslash E_{k}\right) \leq C_{2} 2^{-k} .
\end{gathered}
$$

Let $K$ be a relatively closed subset of the disc $B$. We denote by $S_{+}(B, K)$ the set of bounded subharmonic functions in $B$ vanishing on $K$ and positive and harmonic in $B \backslash K$. We use two standard lemmas.

LEMMA 3.1. Let $\left\{v_{j}\right\}$ be a sequence of uniformly bounded functions from $S_{+}(B, K)$. Let $K$ be regular for the Dirichlet problem in $\mathbb{C} \backslash K$. Then there exists a subsequence which converges pointwise to a function from $S_{+}(B, K)$.

LEMMA 3.2. Let u belong to $S_{+}(2 B, K)$ for some ball $2 B$ of diameter less than 1 and having $\operatorname{cap}(B \cap K)>0$. Then

$$
\sup _{B} u \leq C_{B, K}\left\|\mu_{u}\right\| \text {. }
$$

So as $\left\|\mu_{u_{n_{k}}}\right\| \leq C$ we conclude that $u_{n_{k}}$ are uniformly bounded. We may think that the subsequence in Lemma 3.1 is $\left\{u_{n_{k}}\right\}$ itself; put $u_{0}=$ $\lim _{k \rightarrow \infty} u_{n_{k}}$ in $B$. The convergence is pointwise bounded and so $\mu_{u_{n_{k}}} \rightarrow$ $\mu_{u_{0}}$ weakly. But (3.5) and (3.6) show that $\mu_{u_{n_{k}}} \rightarrow e^{-\gamma+c} d \omega$ weakly. Thus $d \mu_{u_{0}}=e^{-\gamma+c} d \omega$ and $u_{0} e^{c}$ satisfies (3.2).

Now the construction of $\tau$ follows word for word the construction in $[\mathrm{LyV}],[\mathrm{BV}]$. We sketch it here for the sake of completeness. We consider a component $B_{\theta}$ of $f^{-n} B$ and define $\tau$ on it as follows:

$$
\tau(z):=\frac{1}{d^{n}} u\left(f^{n} z\right)
$$

In $[\mathrm{LyV}]$ or $[\mathrm{BV}]$ it is shown that $\tau$ (or its symmetrization) does not depend on $\theta$ or $n$, that is, if $B_{\theta_{1}} \cap B_{\theta_{2}} \neq \emptyset$ then $\tau$ (or its symmetrization) is the same on this intersection. This follows quite easily from (3.2). Now $\tau$ is defined on the set $O=\bigcup f^{-n} B$. Using (2.2) it is easy to extend it to $U$. Theorem 1 is therefore proved.

Proof of Theorem 2. The circular model of Douady and Hubbard associates with a polynomial-like map $f$ an expanding real analytic endomorphism $F$ of degree $d$ of the unit circle $\mathbb{T}$. If $f$ is a polynomial, then 
$F: z \rightarrow z^{d}$. Polynomial-like maps are called externally equivalent if the corresponding endomorphisms are analytically conjugate.

Theorem B $([\mathrm{DH}])$. Let $f$ be a polynomial-like map of degre $d$. Then $f$ is conformally equivalent to a polynomial if and only if it is externally equivalent to $z \rightarrow z^{d}$.

An automorphic harmonic function $\tau$ built in Section 3 gives this external equivalence easily. This is done in $[\mathrm{LyV}]$, but we will sketch the proof here. Let $F$ be a Douady-Hubbard external map for $f$. Then $F: A_{1} \rightarrow A_{2}$ is an analytic covering of degree $d$ between annuli $A_{1}, A_{2}$ with $\bar{A}_{1} \subset A_{2}$ and $\mathbb{T} \subset \partial A_{1} \cap \partial A_{2}$. Given the $f$-automorphic harmonic function $\tau$ we can pull it on $A_{1}$, thus constructing an $F$-automorphic harmonic function $T$ on $A_{1}$ with $T(F z)=d T(z)$. Then $T$ vanishes on the unit circle. We can extend $T$ through the circle by reflection to obtain a subharmonic function with $\Delta T=\varrho d \sigma$, where $\sigma$ denotes the Lebesgue measure on the circle and $\varrho$ is real analytic. But the automorphic equation shows that the Riesz measure $\Delta T$ of $T$ is a maximal measure for $F$. Thus $m_{F}$ is equivalent to the Lebesgue measure and a theorem of Shub and Sullivan [SS] finishes the proof by showing that $F$ is analytically equivalent to $z \rightarrow z^{d}$.

4. Questions. We finish by formulating two questions:

1. Can one use the technique of $[\mathrm{MR}]$ to prove the theorems above?

2. Given a polynomial-like map $f$ and its external map $F: \mathbb{T} \rightarrow \mathbb{T}$, is it true that the harmonic measure on $J_{f}$ is transferred to a measure absolutely continuous with respect to Lebesgue measure on the circle?

Positive answers would give an easy proof of Theorem 2. But it will not be possible to use it for Theorem 1 without extending the Douady-Hubbard external model to GPL.

5. Nonlinearizability of harmonic measure. The method used for the proof of Theorem 1 shows that the logarithm of the Jacobian of harmonic measure $\phi$ can satisfy $\phi-\psi=\gamma \circ f-\gamma$ with continuous $\psi$ having only finitely many values only if $\psi$ is actually a constant function, equal to $\log d$, and $f$ is conformally maximal.

\section{References}

[B] Z. Balogh, Rigidity of harmonic measure on totally disconnected fractals, preprint, Michigan State University, April 1995.

[BPV] Z. Balogh, I. Popovici and A. Volberg, Conformally maximal polynomial-like dynamics and invariant harmonic measure, preprint, Uppsala Univ., U.U.D.M. Report 1994:32. 
[BV] Z. Balogh et A. Volberg, Principe de Harnack à la frontière pour des répulseurs holomorphes non récurrents, C. R. Acad. Sci. Paris 319 (1994), 351-354.

[Br] H. Brolin, Invariant sets under iteration of rational functions, Ark. Mat. 6 (1965), 103-145.

[DH] A. Douady and F. Hubbard, On the dynamics of polynomial like mappings, Ann. Sci. Ecole Norm. Sup. 18 (1985), 287-345.

[Fo] S. R. Foguel, The Ergodic Theory of Markov Processes, Math. Stud. 21, Van Nostrand, New York, 1969.

[FLM] A. Freire, A. Lopes and R. Mañé, An invariant measure for rational maps, Bol. Soc. Brasil Mat. 14 (1983), 45-62.

[Lo] A. Lopes, Equilibrium measure for rational functions, Ergodic Theory Dynam. Systems 6 (1986), 393-399.

[Ly1] M. Lyubich, Entropy of the analytic endomorphisms of the Riemann sphere, Funktsional. Anal. i Prilozhen. 15 (4) (1981), 83-84 (in Russian).

[Ly2] -, Entropy properties of rational endomorphisms of the Riemann sphere, Ergodic Theory Dynam. Systems 3 (1983), 351-386.

[LV] M. Yu. Lyubich and A. Volberg, A comparison of harmonic and maximal measures on Cantor repellers, J. Fourier Anal. Appl. 1 (1995), 359-379.

[M] R. Mañé, On the uniqueness of the maximizing measure for rational maps, Bol. Soc. Brasil. Mat. 14 (1983), 27-43.

[MR] R. Mañé and L. F. da Rocha, Julia sets are uniformly perfect, Proc. Amer. Math. Soc. 116 (1992), 251-257.

[SS] M. Shub and D. Sullivan, Expanding endomorphisms of the circle revisited, Ergodic Theory Dynam. Systems 5 (1985), 285-289.

[Z1] A. Zdunik, Parabolic orbifolds and the dimension of the maximal measure for rational maps, Invent. Mat. 99 (1990), 627-649.

[Z2] - Invariant measure in the class of harmonic measures for polynomial-like mappings, preprint 538, Inst. Math., Polish Acad. Sci., 1995.

Department of Mathematics

Michigan State University

East Lansing, Michigan 48824

U.S.A.

E-mail: irina@math.msu.edu volberg@math.msu.edu

Received 28 April 1995;

in revised form 29 December 1995 